\title{
Micro Reverse Monte Carlo Approach to EXAFS Analysis
}

\author{
Keisuke Fujikawa, Hiroko Ariga, Satoru Takakusagi, Hiromitsu Uehara, Tadashi Ohba, and Kiyotaka Asakura* \\ Catalysis Research Center, Hokkaido University, Kita 21-10, Sapporo 001-0021, Japan
}

(Received 13 February 2014; Accepted 19 April 2014; Published 5 July 2014)

\begin{abstract}
We have developed a new Reverse Monte Carlo code, called the micro Reverse Monte Carlo (m-RMC), which is applicable to structure analysis of nanomaterials and surface species. In the m-RMC, Reverse Monte Carlo is applied to an ensemble of replica files, each of which contains one molecule or one small cluster, because the Extended X-ray Absorption Fine Structure (EXAFS) is sensitive to short-range structures and has negligible interaction between molecules or clusters. We apply the m-RMC to face-centered cubic metals $(\mathrm{Cu}, \mathrm{Pd}$, and $\mathrm{Pt})$ and discuss the advantages, validation, and problems of the m-RMC. The bond distance and some cumulant coefficients can be determined from the EXAFS using m-RMC. Some 50-100 replica files are sufficient to reproduce the EXAFS oscillations and radial distributions. The bond distance can be determined, including the asymmetric distributions, by m-RMC. We also apply m-RMC to $\alpha-\mathrm{MoO}_{3}$ and $\mathrm{Au}$ clusters. The m-RMC analysis of $\mathrm{MoO}_{3}$ shows that three radial distribution peaks appear corresponding to three types of Mo-O bonds. The m-RMC analysis of Au cluster indicates the presence of $\mathrm{Au}_{55}$ cuboctahedral structure with $\mathrm{Au}-\mathrm{Au}$ distance at $0.288 \mathrm{~nm}$. We obtain a $3 \mathrm{D}$ image of $\mathrm{Au}_{55}$ nanocluster from the unified file. The m-RMC method can be applied to the analysis of the EXAFS for chemical systems with appropriate care. [DOI: 10.1380/ejssnt.2014.322]
\end{abstract}

Keywords: Computer simulations; X-ray absorption spectroscopy; Palladium; Platinum; Copper

\section{INTRODUCTION}

The Extended X-ray Absorption Fine Structure (EXAFS) is one of the most important tools for local structure analysis of nanomaterials and surface species [1]. Polarization dependent EXAFS is applied to metal species dispersed on the surface of oxide single crystal as a model supported catalyst and has provided three dimensional structures of surface metal species and the interaction between metal and surface [2].

However, the EXAFS method has several limitations. One weakness lies in the analysis of a system with an asymmetric radial distribution, which is often found in an amorphous structure [3, 4], where the coordination numbers and bond lengths are less reliable when the data are analyzed with a conventional EXAFS equation, because this assumes Gaussian or symmetric radial distribution curves.

Several methods have been proposed to treat the asymmetric atomic distribution such as cumulant expansion, regularization method and genetic algorithm [5-8]. In the cumulant expansion method [5], the EXAFS oscillation from one coordination shell is expressed as:

$$
\begin{aligned}
\chi(k)= & S_{0} \frac{N F_{\text {eff }}(k, r)}{k r^{2}} e^{\left(-2 k^{2} C_{2}+\frac{2}{3} C_{4} k^{4}-\cdots\right)} \\
& \times \sin \left(2 k r+\phi_{\text {eff }}(k, r)-\frac{4}{3} C_{3} k^{3}+\cdots\right),
\end{aligned}
$$

where $S_{0}, N, r$, and $k$ are the inelastic reduction factor, coordination number, bond distance, and wave number of the photoelectron, respectively; $F_{\text {eff }}$ and $\phi_{\text {eff }}$ are the effective backscattering amplitude and phase shift functions derived from theoretical calculations such as the FEFF code [9]; and $C_{2}, C_{3}$, and $C_{4}$ are the 2nd, 3rd, and 4 th cumulant coefficients [5]. Here, $C_{2}$ is equal to the square of the Debye-Waller factor or the relative meansquare displacement and $C_{3}$ reflects the asymmetric distribution and is related to the thermal expansion [10]. $C_{4}$

*Corresponding author: askr@cat.hokudai.ac.jp describes the symmetric deviation from the Gaussian distribution [11]. They are directly related to anharmonic potential through path integral method [12-14]. If the EXAFS of one coordination shell with one type of coordinating element can be extracted from the total EXAFS oscillation by Fourier filtering, then the ratio method can be applied, which provides us with reliable cumulant coefficient [5, 15, 16]. However, the cumulant expansion analysis has two limitations. One is that it requires more fitting parameters than the conventional EXAFS analysis and the other is that there is no guarantee for the convergence of the cumulant expansion in a largely disordered system [4].

The other method is the reverse Monte Carlo (RMC) method [17-19]. The RMC method is used to solve an inverse problem in neutron and X-ray diffraction [17]. The EXAFS is reproduced by a large number of atoms, $N,\left(N=10^{2}-10^{3}\right)$ confined in a large cell with periodic boundary conditions. The $N$ atoms are allowed to walk randomly to reduce the residual ( $R$-factor) between the calculated and experimental EXAFS spectra in a Metropolis algorithm. After many cycles of random walks, the $R$-factor has negligible changes, which is identified as equilibrium in this paper. When equilibrium has been reached, we can derive the radial distribution function (RDF) and several structure parameters $\left(r, C_{2}\right.$, and $C_{3}$ ) from the statistical analysis of the RDF [18]. The RMC uses a three-dimensional real-space structure and, thus, the multiple scattering paths are automatically included with the assistance of an EXAFS calculation program such as FEFF [9]. The RMC is mainly applied to the EXAFS analysis of condensed matters, e.g., amorphous $\mathrm{Si}$ [18], liquid $\mathrm{KPb}$ [20], undercooled $\mathrm{Cu}$ [21], water [22], $\mathrm{Li}_{2} \mathrm{Fe}_{2} \mathrm{O}_{3}$ [23], and $\mathrm{Ca}(\mathrm{Zr}, \mathrm{Ti}) \mathrm{O}_{3}$ solid solutions [24].

In contrast, RMC is rarely applied to discrete systems composed of molecules and small clusters, in which chemists and nanoscientists are interested. Although some examples were found in the application of the conventional RMC analysis to liquid molecular systems such as water [22, 25], a large number of independent molecules or clusters are present in a large cell, though EXAFS is mostly determined by the structure inside the components 
(molecules or clusters) and the contribution to EXAFS from the interaction between the components (molecules and clusters) is negligible. In this sense, the conventional $\mathrm{RMC}$ is inefficient. In this paper, we propose another $\mathrm{RMC}$, called a micro reverse Monte Carlo (m-RMC), which is designed for the discrete system. The m-RMC utilizes a small file without periodic boundaries, in which only one molecule or one cluster is present, so that we completely neglect the interaction between molecules or clusters. However, one file is insufficient to represent the entire system, so many replica files are prepared. The $\mathrm{RMC}$ procedure is applied to each replica file while the EXAFS oscillation is calculated for each file and averaged in order to compare the calculated EXAFS oscillation with the experimental data. After the residual between the calculated and experimental values achieves equilibrium, we derive the RDF from the ensemble of replica files. Each file is expected to be a virtual model of the real molecule or cluster, which may provide us with a threedimensional structure. The merit of m-RMC method is as follows.

(1) The neglect of the intermolecular interaction increases the calculation speed.

(2) We can recognize a molecule or a small cluster directly from each file.

(3) It is easy to combine m-RMC with the local force field calculation.

The concept of m-RMC was already published by Di Cicco et al. [26], who applied it to molecular $\mathrm{Br}_{2}$. However, there has been no systematic study of the m-RMC method. In this research, we apply the m-RMC method to face-centered cubic (fcc) metal foils, particularly Pd, in order to validate the method. $\mathrm{Cu}, \mathrm{Pd}$ and $\mathrm{Pt}$ foils have advantages that we can measure good EXAFS data and can compare the results with the literature. Many physical properties of fcc metals are available in the literature, enabling us to measure the EXAFS more precisely. We would like to answer the following questions:

1. Is the m-RMC applicable to EXAFS analysis?

2. How many files are necessary to determine the structure?

3. What kind of information do we derive from the $\mathrm{m}-\mathrm{RMC}$ ?

4. Can each file be a virtual model structure of the real system?

In the final part, we briefly report the results on mRMC applied to $\mathrm{MoO}_{3}$, a highly distorted oxide and $\mathrm{Au}$ nanocluster.

\section{2. CALCULATION AND EXPERIMENT}

\section{A. m-RMC method and algorithm}

In principle m-RMC method follows the conventional RMC procedure. In m-RMC each molecule or cluster was divided into independent file. The initial model structure was assumed, and then the initial configurations were copied to replica files. The necessary number of replica files was discussed below. Each $\chi_{\text {cal }}^{i}(k)$ was calculated using an FEFF8.4 code [9] based on the atomic configuration in each replica file. Consequently multiple scattering effects are included. In the analysis of fcc metals and $\mathrm{MoO}_{3}$ only the central atoms were regarded as $\mathrm{X}$ ray absorbing atoms and the other atoms were treated as electron-scattering atoms for simplicity. In the analysis of $\mathrm{Au}_{55}$ cluster, we treated all $\mathrm{Au}$ as absorbing atoms.

The $\chi_{\text {cal }}^{i}(k)$ was obtained from the initial configuration using the following equation:

$$
\begin{gathered}
\chi_{\text {cal }}^{i}(k)=S_{0} \Sigma_{j}^{N} \frac{F_{\text {eff }}\left(k, r_{i j}\right)}{k r_{i j}^{2}} \sin \left(2 k r_{i j}+\phi_{\text {eff }}\left(k, r_{i j}\right)\right), \\
k=\sqrt{\frac{2 m}{\hbar^{2}}\left(h \nu-E_{0}\right)}
\end{gathered}
$$

where $r_{i} j$ is the bond distance between the $j$-th atoms in the $i$-th file. $E_{0}$ and $m$ were the origin of photoelectron kinetic energy and mass of electron, respectively. $S_{0}$ and $E_{0}$ were obtained from the EXAFS data of the corresponding reference compounds.

The $\chi_{\text {cal }}^{i}(k)$ values were then averaged over all replica files to give the total $\chi_{\text {cal }}(k)$, which was then compared with $\chi_{\exp }(k)$ (from experimental data). The degree of fitting was estimated by an $R$ factor, $R^{2}=\left(\Sigma\left[k^{3} \chi_{\exp }(k)-\right.\right.$ $\left.\left.k^{3} \chi_{\text {cal }}(k)\right]^{2}\right) / \Sigma\left(k^{3} \chi_{\exp }(k)\right)^{2}$, which was minimized by the $\mathrm{m}-\mathrm{RMC}$ method. One atom in each replica file was chosen and then randomly walked to produce a new configuration. In order to avoid the unphysical overlap of surrounding atoms, we used a hard sphere model for each atom with radius of $0.24 \mathrm{~nm}$. If the random walk caused the distance between the two atoms less than this value, the movement was automatically rejected. Otherwise a new $\chi_{\text {cal }}(k)$ was calculated using the new atomic configuration to obtain a new $R$ factor $\left(R_{\text {new }}\right)$. When $R_{\text {new }}$ was smaller than the $R$ factor before the random walk, $R_{\text {old }}$, the new configuration was accepted. When $R_{\text {new }}$ was larger than $R_{\text {old }}$, the Metropolis algorithm was applied, i.e., the new configuration was accepted with a probability of $\exp \left(-\left(R_{\text {new }}^{2}-R_{\text {old }}^{2}\right) / \tau\right)$, where $\tau$ is a controlling parameter to determine the accept/reject ratio.

$R$ was normally varied by approximately $1 \times 10^{-4}$ in the final optimization process, the $\tau$ factor was set at $10^{-6}$ because the number of replica files was typically 100. In order to avoid being trapped at the local minimum and to decrease the calculation time, $\tau$ was first set at a large value of approximately 0.01 and was then gradually decreased to obtain an accept/reject ratio nearly unity. We repeat the random walk procedure on the next atom. After $10^{4}-10^{5}$ steps in the walk, the $R$ factor reached equilibrium. The RDFs and cumulant coefficients from the final configurations are given by the following equations:

$$
\begin{aligned}
r & =\left(C_{1}\right)=\langle r\rangle, \\
C_{2} & =\left\langle(r-\langle r\rangle)^{2}\right\rangle, \\
C_{3} & =\left\langle(r-\langle r\rangle)^{3}\right\rangle .
\end{aligned}
$$



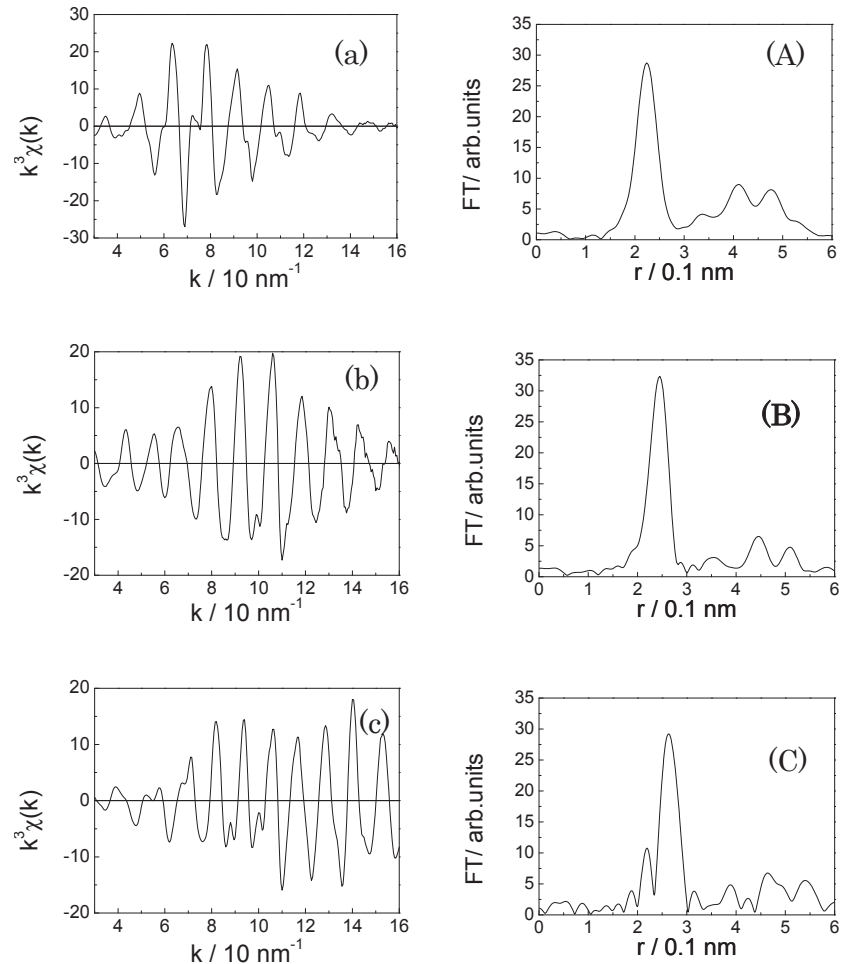

FIG. 1: Experimental data for $k^{3} \chi(k)$ (a-c) and their Fourier transforms (A-C) for $\mathrm{Cu}(\mathrm{a}, \mathrm{A}), \mathrm{Pd}(\mathrm{b}, \mathrm{B})$, and $\mathrm{Pt}(\mathrm{c}, \mathrm{C})$.

\section{B. EXAFS measurements}

The EXAFS spectra were measured in a transmission mode at the Photon Factory (PF) of the Institute of Materials Structure Science, of the High Energy Accelerator Research Organization (KEK-IMSS-PF). The Cu K- ,Au $\mathrm{L}_{3}$ - and $\mathrm{Pt} \mathrm{L}_{3}$-edges were measured at room temperature at the beam line $\mathrm{BL} 12 \mathrm{C}$ of the $\mathrm{PF}$ storage ring $(2.5 \mathrm{GeV}$, $500 \mathrm{~mA}$ ) using a $\mathrm{Si}(111)$ double crystal monochromator. The Mo K- and Pd K-edges were measured at beam line NW10A in the PF-AR (Photon Factory Advanced Ring) operated at $6.5 \mathrm{GeV}, 50 \mathrm{~mA}$ using a $\mathrm{Si}(311)$ double crystal monochromator. The data were processed using REX 2000 [27-29]. Figure 1 shows the experimental data for $k^{3} \chi(k)$ and their Fourier transforms for $\mathrm{Cu}, \mathrm{Pt}$, and $\mathrm{Pd}$. The Fourier transformation range was 30-160 nm ${ }^{-1}$.@ $\mathrm{MoO}_{3}$ was purchased from Wako Chemicals.@@ Au nanoclusters were prepared according to the previously reported way to deposit $\mathrm{Au}\left(\mathrm{PPh}_{3}\right) \mathrm{NO}_{3}$ on $\mathrm{Fe}(\mathrm{OH})_{3}$ followed by $673 \mathrm{~K}$ calcination [30, 31].

\section{RESULTS AND DISCUSSION}

\section{A. m-RMC results for the first shell of the $\mathbf{P d}$ foil}

Since EXAFS oscillation is mainly determined by the first nearest neighbor (FNN), the EXAFS oscillation of the FNN was extracted from the data via inverse Fourier transformation of the first peak in Fig. 1(B), filtered in the range of $0.16-0.31 \mathrm{~nm}$. Since only the FNN was considered, the replica file contains thirteen atoms with one X-ray absorbing atom and 12 FNNs. The calculated
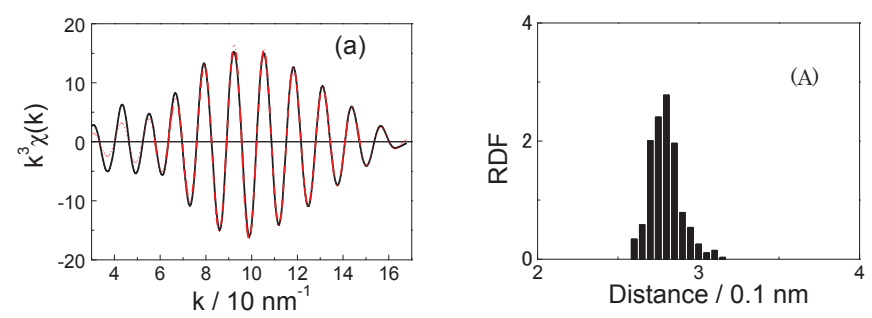

FIG. 2: (a) Comparison of $k^{3} \chi(k)$ calculated using m-RMC (black solid curve) and Fourier filtered data of Pd foil (red broken curve) and (b) the radial distribution curve at the equilibrium after the m-RMC steps.


FIG. 3: Conventional curve fitting results of the Pd K-edge EXAFS data. The black solid curve shows the fitted data and the red broken line shows the experimental data. (a) $C_{3}=0.0 \mathrm{~nm}^{3}$, (b) $C_{3}=2.0 \times 10^{-7} \mathrm{~nm}^{3}$.

$k^{3} \chi_{\text {cal }}(k)$ was obtained by averaging a hundred $k^{3} \chi_{\text {cal }}^{i}(k)$ values of each file. Figure 2(a) presents a comparison of the Fourier-filtered data of the experimental $k^{3} \chi(k)$ for $\mathrm{Pd}$ foil and the calculated values for $k^{3} \chi(k)$ (denoted as $k^{3} \chi_{\exp }(k)$ and $\left.k^{3} \chi_{\text {cal }}(k)\right)$. The $R$ factor at equilibrium was 0.096. Figure 2(b) shows the RDF (histogram) derived from the $\mathrm{m}-\mathrm{RMC}$ results. The RDF was normalized by the number of files so that its integration was equal to the coordination number of FNNs (12). The $r, C_{2}$, and $C_{3}$ values were $0.276 \pm 0.001 \mathrm{~nm},(6.5 \pm 1.0) \times 10^{-5} \mathrm{~nm}^{2}$, and $(2 \pm 1) \times 10^{-7} \mathrm{~nm}^{3}$, respectively (Table I). Figure 3a shows the conventional curve fitting analysis using Eq. (2). The $R$ factor was 0.12 . Figure $3 \mathrm{~b}$ also shows the curve fitting result with optimized $C_{3}=2.0 \times 10^{-7} \mathrm{~nm}^{3}$. The fitting was improved and the results were summarized in Table I.

The measured bond distance was $0.274 \mathrm{~nm}$, a little shorter than the value obtained from the m-RMC. The contraction of the bond distance was due to the asymmetric distribution effect. The asymmetric distribution effect can be corrected by using the cumulant expansion analysis expressed in Eq. (1). In fact, we carried out curve fitting analysis including $C_{3}$ and found that $r=0.275 \pm 0.002 \mathrm{~nm}$, with $C_{2}=(6.5 \pm 1.0) \times 10^{-5} \mathrm{~nm}^{2}$ and $C_{3}=(2 \pm 1) \times 10^{-7} \mathrm{~nm}^{3}$. The m-RMC automatically includes the asymmetry effects.

The $C_{2}$ value of $\mathrm{Pd}$ could be calculated from the Debye

TABLE I: Comparison of m-RMC and curve fitting results.

\begin{tabular}{lcccc}
\hline \hline Method & $\begin{array}{c}r \\
(\mathrm{~nm})\end{array}$ & $\begin{array}{c}C_{2} / 10^{-5} \\
\left(\mathrm{~nm}^{2}\right)\end{array}$ & $\begin{array}{c}C_{3} / 10^{-7} \\
\left(\mathrm{~nm}^{3}\right)\end{array}$ & $R$ \\
\hline m-RMC 100 files & $0.276 \pm 0.001$ & $6.5 \pm 1.0$ & $2 \pm 1$ & 0.096 \\
Curve fitting w/o $C_{3}$ & $0.274 \pm 0.002$ & $6.9 \pm 1.0$ & - & 0.12 \\
Curve fitting w/ $C_{3}$ & $0.276 \pm 0.002$ & $6.5 \pm 1.0$ & $2 \pm 1$ & 0.11 \\
\hline
\end{tabular}





FIG. 4: The normalized radial distribution curves determined by m-RMC for Pd foil: (a) 1 file, (b) 2 files, (c) 10 files, and (d) 50 files, and (e) the corresponding $\mathrm{R}$ factors.

model using the Debye temperature $(275 \mathrm{~K})$ [32]. The value of $C_{2}$ is $6.7 \times 10^{-5} \mathrm{~nm}^{2}$, which is in good agreement with the values of $C_{2}$ obtained from EXAFS.

\section{B. Dependence on the number of files}

One of the most important parameters of the m-RMC method is the number of replica files. Too small a number cannot reproduce the real distribution of atom positions. The more files that are used for the simulation, the more accurate the result will be. However, too large a number of files will increase the computing time. Therefore, an appropriate number of files must be chosen. Here, we discuss how many files are necessary and sufficient to reproduce the oscillation.

The experimental $k^{3} \chi_{\exp }(k)$ values are obtained by inverse Fourier transformation. The Fourier transform range $(\Delta k)$ and the inverse Fourier transform range $(\Delta r)$ were $130(=160-30) \mathrm{nm}^{-1}$ and $0.15 \mathrm{~nm}$, respectively so that the number of degrees of freedom [33] was $N_{\text {free }}=$ $2 \Delta k \Delta r / \pi+2=14.4$. In Eq. (2), the only adjustable parameters were $r_{i j}$ and one file only had twelve adjustable parameters. This is less than $N_{\text {free }}$ and not large enough to accurately reproduce the experimental data with only one file.

Figures 4(a)-(d) show the radial distribution curves derived from different numbers of replica files. Figure 4(e) shows the $R$ factors for each number of files. As ex-
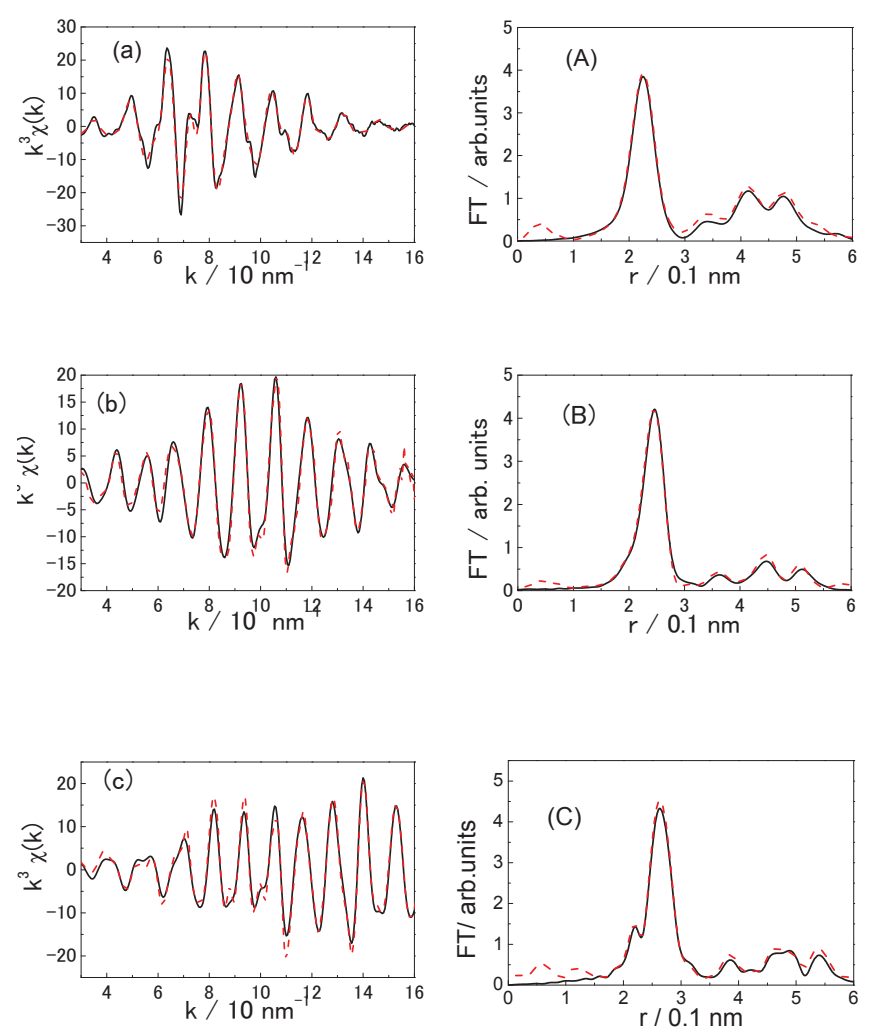

FIG. 5: EXAFS oscillations (left) and their Fourier transforms (right) of (a) $\mathrm{Cu}$, (b) $\mathrm{Pd}$, and (c) Pt foils. The black solid curves and the red broken curves correspond to the m-RMC data and observed data, respectively.

pected, one file was insufficient to accurately reproduce $\chi(k)(R=0.18)$. In the analysis with two files, the $R$ factor was much better $(R=0.13)$. However, looking at the RDF of the two files case shown in Fig. 4(b), the peak is rather rough and split into two, even though the average bond distance and $C_{2}$ were $0.276 \mathrm{~nm}$ and $6.0 \times 10^{-5} \mathrm{~nm}^{2}$, respectively, corresponding well to those obtained with 100 files. Five files improved the $R$ factor to 0.11 . Ten files accurately reproduced the $\chi(k)$ and we acquire a Gaussian-like distribution curve with an $R$ factor of 0.106 . The $r, C_{2}$, and $C_{3}$ are $0.276 \pm 0.001 \mathrm{~nm},(6 \pm 2) \times 10^{-5} \mathrm{~nm}^{2}$, and $(4 \pm 3) \times 10^{-7} \mathrm{~nm}^{3}$, respectively.

When we calculated $\chi(k)$ with more than ten files, we did not greatly improve the $R$ factor (by around 0.10 or a little less). We concluded that at least ten files are necessary and 50-100 files were sufficient for the calculation of the distribution and other structure parameters in the analysis of $\mathrm{Pd}$ foil. The number of adjustable bond lengths was 12 times the number of files, which was between 600 (for 50 files) and 1200 (for 100 files). This number corresponds well to the number of bond lengths necessary for the reproduction of EXAFS oscillation suggested by Di Cicco et al. in their model mimicking the $\mathrm{Br}_{2}$ molecule [21].

\section{C. $\mathrm{Cu}, \mathrm{Pt}$, and Pd fcc foils}

Figure 5 shows the EXAFS data of $\mathrm{Cu}, \mathrm{Pt}$, and $\mathrm{Pd}$ foils measured at room temperature and calculated by $\mathrm{m}$ - 



FIG. 6: RDFs of (a) Cu, (b) Pd, and (c) Pt derived from m-RMC analyses of EXAFS measured at room temperature. The red dotted vertical lines indicate the distances given in the crystallography.

TABLE II: Cumulant parameters derived from m-RMC.

\begin{tabular}{lcccc}
\hline \hline Sample & $\begin{array}{c}r \\
(\mathrm{~nm})\end{array}$ & $\begin{array}{c}C_{2} / 10^{-5} \\
\left(\mathrm{~nm}^{2}\right)\end{array}$ & $\begin{array}{c}C_{3} / 10^{-7} \\
\left(\mathrm{~nm}^{3}\right)\end{array}$ & $R$ \\
\hline $\mathrm{Cu}$ & $0.255 \pm 0.001$ & $9.0 \pm 1.0$ & $5 \pm 3$ & 0.18 \\
$\mathrm{Pd}$ & $0.276 \pm 0.001$ & $6.5 \pm 1.0$ & $2 \pm 1$ & 0.19 \\
$\mathrm{Pt}$ & $0.277 \pm 0.001$ & $4.8 \pm 1.0$ & $1 \pm 1$ & 0.18 \\
\hline \hline
\end{tabular}

RMC. The m-RMC calculations were produced based on 100 replica files with larger clusters composed of 55 atoms. The only absorbing atom was the central atom and the other 54 atoms were scatterers in this calculation. After a large number of random walks of all atoms in 100 files, the $R$ factors were reduced to $0.18,0.19$, and 0.18 , for $\mathrm{Cu}$, $\mathrm{Pt}$, and $\mathrm{Pd}$ foils, respectively, and reached equilibrium. The $R$ factors were a little worse than the results shown above because we included four shells for the calculations in Fig. 5, while the previous calculation was conducted only on the first shell. Fitting was actually a little worse in the higher coordination shells as shown in the Fourier transformation (Fig. 5(A)-(C)).

Figure 6 shows the RDFs determined by m-RMC up to the fourth shell. The parameters in the first shell are given in Table II. The peaks derived from m-RMC agreed well with the positions calculated from the lattice constant. The Debye model provided the $C_{2}$ values as $7.8 \times 10^{-5}$, $6.7 \times 10^{-5}$, and $4.8 \times 10^{-5} \mathrm{~nm}^{2}$ for $\mathrm{Cu}, \mathrm{Pd}$, and Pt, respectively, which agreed well with the $C_{2}$ values obtained from m-RMC analysis. The $C_{3}$ values of $\mathrm{Cu}, \mathrm{Pd}$, and $\mathrm{Pt}$
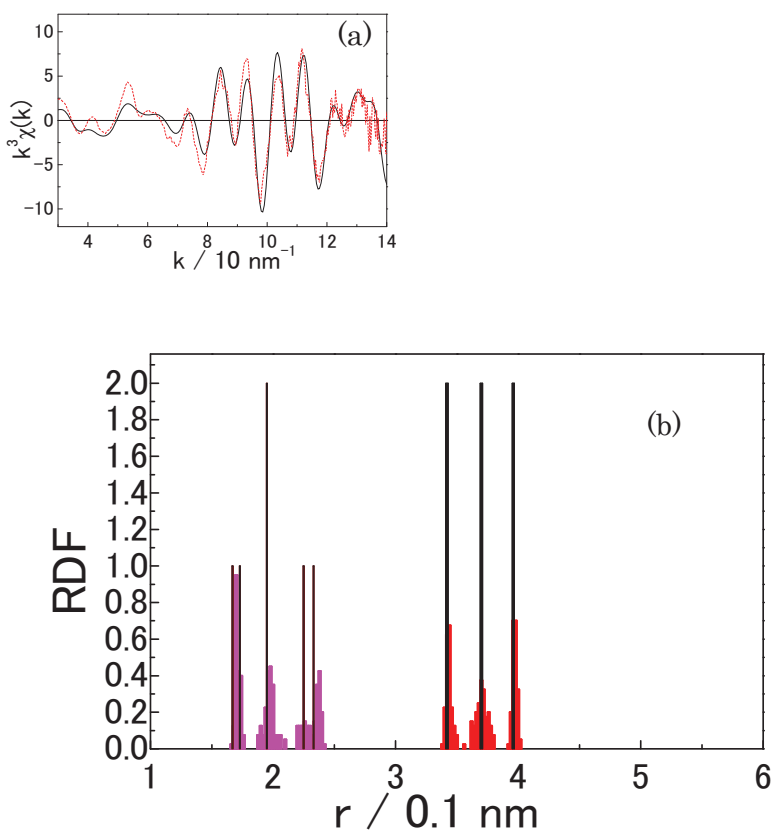

FIG. 7: (a) Comparison of $k^{3} \chi(k)$ calculated using m-RMC (black solid curve) and from experimental data (red broken curve) of $\mathrm{MoO}_{3}$ and (b) the radial distribution curve at the equilibrium after the m-RMC steps. Black vertical bars indicated the distances in the crystallographic data. Purple RDF in the range of $0.1-0.3 \mathrm{~nm}$ indicated $\mathrm{Mo}-\mathrm{O}$ while red $\mathrm{RDF}$ in the range of $0.3-0.45 \mathrm{~nm}$ indicated the Mo-Mo. The Mo-O interaction in this $0.3-0.45 \mathrm{~nm}$ was not shown here in order to simplify the figure.

could be obtained from the thermal expansion $(\alpha)$ using the equation [10]:

$$
C_{3}=2 \alpha r T C_{2},
$$

yielding $2.3 \times 10^{-7}, 1.3 \times 10^{-7}$, and $0.7 \times 10^{-7} \mathrm{~nm}^{3}$ for $\mathrm{Cu}, \mathrm{Pd}$, and $\mathrm{Pt}$, respectively. These were found in the literature as $1.3 \times 10^{-7}, 0.90 \times 10^{-7}$, and $1.1 \times 10^{-7} \mathrm{~nm}^{3}$ for $\mathrm{Cu}[34], \mathrm{Pd}[16,35]$, and $\mathrm{Pt}[36]$, respectively, which correspond well to the values given in Table II. Consequently, the m-RMC method can reproduce the structure parameters, such as the bond distance and the $C_{2}$ and $C_{3}$ cumulants.

\section{Preliminary applications to $\mathrm{MoO}_{3}$ and $\mathrm{Au}$ nanoparticles}

The m-RMC method was applied to the $\mathrm{MoO}_{3}$ and $\mathrm{Au}$ nanoparticle. Preliminary results are presented here to discuss the future directions. First example is $\mathrm{MoO}_{3}$. Mo oxide nanocluster and surface thin layer are catalytically important oxides for the selective oxidation reactions [3740]. Since $\mathrm{MoO}_{3}$ has an anisotropic structure with 5 different bond lengths at $0.167,0.1760 .195 \times 2,0.225$ and $0.233 \mathrm{~nm}[41,42]$, it is difficult to determine the bond distances correctly by conventional EXAFS analysis [43], though such anisotropic structure has an important role in the selective oxidation reaction [44-46]. We carried out the m-RMC analysis on $\mathrm{MoO}_{3}$. Figure $7(\mathrm{a})$ shows the comparison between the $k^{3} \chi(k)$ calculated by the mRMC method and the observed one. The frequency of 



FIG. 8: (a) Comparison of $k^{3} \chi(k)$ calculated using m-RMC (black solid curve) and from experimental data (red broken curve) of Au nanoparticles and (b) the radial distribution curve at the equilibrium after the m-RMC steps.

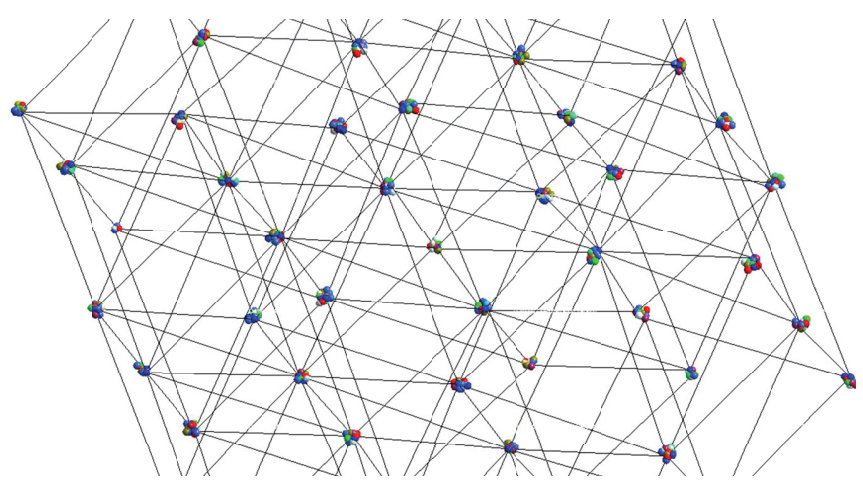

FIG. 9: The cluster image obtained from m-RMC for Au nanocluster. The all $\mathrm{m}-\mathrm{RMC}$ replica files were unified to one file and all atoms were plotted. Each overlapping small balls indicated the presence of $\mathrm{Au}$ atoms in different replica files.

the oscillation was well reproduced. Figure $7(\mathrm{~b})$ shows the radial distribution functions. The vertical bars in the figure indicated the $\mathrm{Mo}-\mathrm{O}$ positions determined by the crystallography. There were three peaks appearing at the corresponding Mo-O positions to those of the crystal data in the range of $0.15-0.3 \mathrm{~nm}$. The first peak was composed of two Mo-O bondings at 0.167 and $0.173 \mathrm{~nm}$. It was difficult to distinguish the two Mo-O bonds owing to the finite $k$ range (30-140 $\mathrm{nm}^{-1}$ ) which gave the bond distance resolution as $0.014 \mathrm{~nm}=\pi / 2 \Delta k$. Thus a peak in m-RMC simulation appeared at $0.170 \mathrm{~nm}$ just at the average position of the two Mo-O bonds. In the present m-RMC simulation the second peak appeared at $0.197 \mathrm{~nm}$ shifted from the $\mathrm{MoO}_{3}$ crystal data by $0.002 \mathrm{~nm}$. A broad peak was observed around $0.237 \mathrm{~nm}$ which corresponded to the long Mo-O bonds at 0.225 and $0.235 \mathrm{~nm}$. The deviation in the position and broadening were due to the smaller contribution of the Mo-O bond to XAFS oscillation. Actually in the polarization dependent XAFS measurement of $\mathrm{MoO}_{3}$, the peak of $\mathrm{Mo}-\mathrm{O}$ at $0.225 \mathrm{~nm}$ was less than a half of $0.173 \mathrm{~nm}$ peak $[42,43]$. Figure $7(\mathrm{~b})$ also shows the Mo-Mo radial distribution function. Three peaks appeared at $0.343 \mathrm{~nm}, 0.370 \mathrm{~nm}$ and $0.396 \mathrm{~nm}$ corresponding to the three Mo-Mo distances in $\mathrm{MoO}_{3}$ crystal as shown in Fig. 7(b). Note that there was a broader peak at $0.370 \mathrm{~nm}$ than the other two Mo-Mo peaks. The direction of $0.370 \mathrm{~nm}$ peak corresponded to [001] in Pbnm space group which showed smallest thermal expansion and compression [47]. Further studies using polarization and temperature dependence measurement are interesting to reveal the anisotropic behavior of Mo-Mo lattice interaction.

In the next example we tested $\mathrm{Au}$ nanoparticles deposited on $\mathrm{Fe}(\mathrm{OH})_{3}$. Au nanoparticle drew attentions because of its high low temperature $\mathrm{CO}$ oxidation activity $[48,49]$. We constructed $\mathrm{Au}_{55}$ cuboctahedron cluster since the conventional EXAFS gave the 7.7 as coordination number. Then the replica files were optimized by the m-RMC method. In this case all $\mathrm{Au}$ atoms in the file were regarded as x-ray absorbing atoms and EXAFS oscillations were calculated for all of them. $5 \times 10^{4} \mathrm{cy}-$ cles of FEFF calculations were applied and the simulated curve was obtained as shown in Fig. 8(a). The radial distribution was obtained as shown in Fig. 8(b). The bond distance, coordination number and $C_{2}$ for the first shell were $0.288 \mathrm{~nm}, 7.7$ and $3.1 \times 10^{-5} \mathrm{~nm}^{2}$, respectively. The bond distance was almost the same as the one in the crystal. We are now developing a program by changing the number of $\mathrm{Au}$ atoms in each cluster during the m-RMC process to obtain the size distribution.

\section{E. The potential of m-RMC and conclusions}

In this work, we have discussed the potential of the m-RMC method by comparing the experimental and calculated EXAFS oscillations of $\mathrm{Cu}, \mathrm{Pd}$, and Pt fcc metals. We raised four questions and tried to answer them:

1. Is the m-RMC method applicable to EXAFS analysis?

The m-RMC method can be applicable to the analysis of EXAFS and can determine the bond length exactly by including the correction of the asymmetric distribution.

2. How many files are necessary to determine the structure?

At least ten files are required to reproduce the data and 50-100 files are sufficient for the full calculation in the case of $\mathrm{Pd}$ foil.

3. What kind of information do we derive from the $\mathrm{m}$ RMC? 
We obtain the RDF and:

$$
\begin{aligned}
r & =\left(C_{1}\right)=\langle r\rangle, \\
C_{2} & =\left\langle(r-\langle r\rangle)^{2}\right\rangle, \\
C_{3} & =\left\langle(r-\langle r\rangle)^{3}\right\rangle .
\end{aligned}
$$

We can of course calculate $C_{4}$ but will still have a large error compared to the absolute value of $C_{4}$, which is very small in the fcc metal. Therefore, we have not discussed it in the above work. This might play an important role in a more disordered system.

4. Can each file be a virtual model of the real system? We cannot currently confirm this because of the following reasons.

(1) $R_{i}$ ( $R$-factor of $i$-th file) is larger than $R$ (overall R-factor determined from the m-RMC method) because there is no necessity that each $\chi_{\text {cal }}^{i}(k)$ should converge to the $\chi_{\exp }(k)$. Actually $R_{i}$ values of some replica files were more than 1 even after the over-all $R$ was nearly 0.1 . We could apply some regulation on each $R_{i}$ (For example, $R_{i}$ should be less than the minimum value that one replica file could achieve, namely 0.18 as shown in Sec III.B) and we had the overall $R$-factor nearly 0.1 . However each replica file does not completely reproduce the results because of small number of atomic pair available in each file. The unification of all files would give more reasonable $3 \mathrm{D}$ image with distributions. Figure 9 shows $\mathrm{Au}_{55}$ nanocluster image where every site contains $\mathrm{Au}$ atoms in all replica files. Since each replica file has an identical structure, it is easy to find the corresponding atomic coordinates from independent files.
(2) In the m-RMC analysis of the fcc metal discussed above where only the EXAFS oscillation from the central atom was taken into account, we got little information about bond angles because the multiple scattering effect in the EXAFS region was usually smaller than the single scattering except collinear case. This point could be improved in the analysis of $\mathrm{Au}_{55}$ nanocluster where correlations of all $\mathrm{Au}$ atoms were included. The force field constrain will be helpful $[25,50-52]$. In this work we have included hard sphere interaction to avoid unphysical overlap of atoms. Another remedy is to combine the m-RMC with the polarization dependent XAFS analysis. As mentioned in introduction, polarization dependent XAFS can be obtained from metal species on the single crystal oxide surface which determined the bond angle and $3 \mathrm{D}$ structure [53-57]. The mRMC method will provide the distributions of the bond angle.

\section{Acknowledgments}

This work was financially supported by the "Development for the implementation of a Polymer Membrane Fuel Cell" project of the New Energy and Industrial Technology Development Organization (NEDO) and the Grant-in-Aid for Scientific Research on Innovative Areas "Nano Informatics" (grant number 25106010) from JSPS (Japan Society for the Promotion of Science). We thank Mr. Shinsuke Sato and Mr. Naoki Watanabe for their first preliminary investigations of m-RMC.
[1] G. Bunker, Introduction to XAFS: A Practical Guide to X-ray Absorption Fine Structure Spectroscopy (Cambridge University Press, Cambridge, 2010).

[2] K. Asakura, Catalysis, Vol. 24, Eds. J. J. Spivey and M. Gupta (RSC publishing, Cambridge, 2012), p. 281.

[3] E. D. Crozier, EXAFS Spectroscopy, Eds. B. K. Teo and D. C. Joy (Springer US, 1981), p. 89.

[4] E. D. Crozier, J. J. Rehr, and R. Ingalls, EXAFS, XANES and SEXAFS, Eds. D. C. Koningsberger and R. Prins (John Wiley \& Sons, New York, 1988), p. 373.

[5] G. Bunker, Nucl. Instrum. Methods 207, 437 (1983).

[6] Y. A. Babanov, V. V. Vasin, A. L. Ageev, and N. V. Ershov, Phys. Stat. Solidi 105, 747 (1981).

[7] A. Kuzmin and J. Purans, J. Phys. C 12, 1959 (2000).

[8] N. Dimakis and G. Bunker, Biophys. J. 91, L87 (2006).

[9] J. J. Rehr and R. C. Albers, Rev. Mod. Phys. 72, 621 (2001).

[10] A. I. Frenkel and J. J. Rehr, Phys. Rev. B 48, 585 (1993).

[11] G. Dalba and P. Fornasini, J. Synchrotron Rad. 4, 243 (1997).

[12] T. Yokoyama, Phys. Rev. B 57, 3423 (1998).

[13] T. Fujikawa and T. Miyanaga, J. Phys. Soc. Jpn. 62, 4108 (1993).

[14] T. Miyanaga and T. Fujikawa, J. Phys. Soc. Jpn. 63, 1036
(1994).

[15] T. Yokoyama, K. Asakura, Y. Iwasawa, and H. Kuroda, J. Phys. Chem. 93, 8323 (1989).

[16] T. Yokoyama, T. Satsukawa, and T. Ohta, Jpn. J. Appl. Phys. 28, 1905 (1989).

[17] R. L. McGreevy and L. Pusztai, Mol. Simul. 1, 359 (1988).

[18] S. J. Gurman and R. L. McGreevy, J. Phys. Condens. Matter 2, 9463 (1990).

[19] R. L. McGreevy, J. Phys. Condes. Matter 13, R877 (2001).

[20] W. Bras, R. Xu, J. D. Wicks, F. van der Horst, M. Oversluizen, R. L. McGreevy, and W. van der Lugt, Nucl. Instrum. Methods A 346, 394 (1994).

[21] A. Di Cicco, A. Trapananti, S. Faggioni, and A. Filipponi, Phys. Rev. Lett. 91, 135505 (2003).

[22] K. T. Wikfeldt, M. Leetmaa, A. Mace, A. Nilsson, and L. G. M. Pettersson, J. Chem. Phys. 132, 104513 (2010).

[23] K. Németh, K. W. Chapman, M. Balasubramanian, B. Shyam, P. J. Chupas, S. M. Heald, M. Newville, R. J. Klingler, R. E. Winans, and J. D. Almer, J. Chem. Phys. 136, 074105 (2012).

[24] V. Krayzman, I. Levin, and M. G. Tucker, J. Appl. Crystallogr. 41, 705 (2008).

[25] O. Gereben and L. Pusztai, J. Comput. Chem. 33, 2285 
(2012).

[26] A. Di Cicco and A. Trapananti, J. Phys. C 17, S135 (2005).

[27] K. Asakura, X-ray Absorption Fine Structure for Catalysts and Surfaces, Vol. 2, Ed. Y. Iwasawa (World Scientific, Singapore, 1996), p. 33.

[28] T. Taguchi, T. Ozawa, and H. Yashiro, Physica Scripta T115, 205 (2005).

[29] T. Taguchi, AIP Conf. Proc. 882, 162 (2006).

[30] Y. Yuan, A. P. Kozlova, K. Asakura, H. Wan, K. Tsai, and Y. Iwasawa, J. Catal. 170, 191 (1997).

[31] A. I. Kozlov, A. P. Kozlova, K. Asakura, Y. Matsui, T. Kogure, T. Shido, and Y. Iwasawa, J. Catal. 196, 56 (2000).

[32] C. Kittel, Introduction to Solid State Physics, 7th ed. (John Wiley \& Sons, New York, 1996).

[33] E. Stern, Phys. Rev. B 48, 9825 (1993).

[34] S. A. Beccara, G. Dalba, P. Fornasini, R. Grisenti, F. Pederiva, A. Sanson, D. Diop, and F. Rocca, Phys. Rev. B 68, 140301 (2003).

[35] T. Yokoyama and T. Ohta, Jpn. J. Appl. Phys. 29, 2052 (1990).

[36] R. Giulian, L. L. Araujo, P. Kluth, D. J. Sprouster, C. S. Schnohr, G. J. Foran, and M. C. Ridgway, J. Phys. C 21, $155302(2009)$.

[37] M. A. Banares, H. Hu, and I. E. Wachs, J. Catal. 150, 407 (1994).

[38] T. Machej, J. Haber, A. M. Turek, and I. E. Wachs, Appl. Catal. 70, 115 (1991).

[39] T. Ushikubo, K. Oshima, A. Kayou, M. Vaarkamp, and M. Hatano, J. Catal. 169, 394 (1997).

[40] S. Albonetti, F. Cavani, and F. Trifiro, Catal. Rev. 28, 413 (1996).

[41] L. Kihlborg, Arkiv Kemi 21, 357 (1963).

[42] W. J. Chun, K. Ijima, Y. Ohminami, S. Suzuki, and K. Asakura, J. Synchrotron Rad. 11, 291 (2004).
[43] K. Ijima, Y. Ohminami, S. Suzuki, and K. Asakura, Topics in Catal. 18, 125 (2002).

[44] J. Ziolkowski, J. Catal. 80, 263(1983).

[45] S. Sato, H. Niimi, S. Suzuki, W.-J. Chun, K. Irokawa, H. Kuroda, and K. Asakura, Chem. Lett. 33, 558 (2004).

[46] M. G. Moula, S. Sato, K. Irokawa, H. Niimi, S. Suzuki, K. Asakura, and H. Kuroda, Bull. Chem. Soc. Jpn. 81, 836 (2008).

[47] H. Negishi, S. Negishi, Y. Kuroiwa, N. Sato, and S. Aoyagi, Phys. Rev. B 69, 064111 (2004).

[48] M. Haruta, T. Kobayashi, H. Sano, and N. Yamada, Chem. Lett. 16, 405 (1987).

[49] M. Haruta, S. Tsubota, T. Kobayashi, H. Kageyama, M. J. Genet, and B. Delmon, J. Catal. 144, 175 (1993).

[50] G. Opletal, T. C. Petersen, B. O'Malley, I. K. Snook, D. G. McCulloch, and I. Yarovsky, Comp. Phys. Commun. 178, 777 (2008).

[51] H. Morita, S. Kohara, and T. Usuki, J. Mol. Liq. 147, $182(2009)$.

[52] A. K. Soper, Phys. Rev. B 72, 104204 (2005).

[53] Y. Koike, K. Ijima, W. J. Chun, H. Ashima, T. Yamamoto, K. Fujikawa, S. Suzuki, Y. Iwasawa, M. Nomura, and K. Asakura, Chem. Phys. Lett. 421, 27 (2006).

[54] W. J. Chun, Y. Koike, H. Ashima, K. Kinoshita, K. Ijima, K. Fujikawa, S. Suzuki, M. Nomura, Y. Iwasawa, and K. Asakura, Chem. Phys. Lett. 470, 99 (2009).

[55] W. J. Chun, Y. Koike, K. Ijima, K. Fujikawa, H. Ashima, M. Nomura, Y. Iwasawa, and K. Asakura, Chem. Phys. Lett. 433, 345 (2007).

[56] S. Takakusagi, H. Nojima, H. Ariga, H. Uehara, K. Miyazaki, W.-J. Chun, Y. Iwasawa, and K. Asakura, Phys. Chem. Chem. Phys. 15, 14060 (2013).

[57] K. Asakura, S. Takakusagi, H. Ariga, W.-J. Chun, S. Suzuki, Y. Koike, H. Uehara, K. Miyazaki, and Y. Iwasawa, Faraday Disc. 162, 165 (2013). 